Itinéraires Itinéraires

Littérature, textes, cultures

2018-1| 2018

Le manifeste à travers les arts : devenirs d'un genre indiscipliné

\title{
Les formats-manif de l'industrie de la mode : stéréotypes et trivialité
}

Manifesto-Patterns of the Fashion Industry: Stereotypes and Triviality

\section{Eleni Mouratidou}

\section{(2) OpenEdition}

\section{Journals}

Édition électronique

URL : http://journals.openedition.org/itineraires/4273

DOI : $10.4000 /$ itineraires. 4273

ISSN : 2427-920X

Éditeur

Pléiade

Référence électronique

Eleni Mouratidou, «Les formats-manif de l'industrie de la mode : stéréotypes et trivialité », Itinéraires [En ligne], 2018-1 | 2018, mis en ligne le 15 septembre 2018, consulté le 16 mai 2020. URL : http:// journals.openedition.org/itineraires/4273 ; DOI : https://doi.org/10.4000/itineraires.4273

Ce document a été généré automatiquement le 16 mai 2020.

\section{cc) $(9)$}

Itinéraires est mis à disposition selon les termes de la licence Creative Commons Attribution - Pas d'Utilisation Commerciale - Pas de Modification 4.0 International. 


\title{
Les formats-manif de l'industrie de la mode : stéréotypes et trivialité
}

\author{
Manifesto-Patterns of the Fashion Industry: Stereotypes and Triviality
}

\author{
Eleni Mouratidou
}

1 Loin des manifestes portant sur la mode en tant qu'acte de création et pratique vestimentaire, les formats-manif que je me propose ici d'analyser ne désignent pas la mode en tant que processus créatif mais en tant que "créativité managériale ${ }^{1}$ " (Barrère et Santaga 2005). Ils relèvent de stratégies communicationnelles visant la promotion d'une ou plusieurs entités commerciales de cette industrie et n'émanent pas de réflexions collectives ou individuelles qui seraient dépourvues d'enjeux et d'intérêts économiques directs. Bien que la mode ait déjà fait l'objet de déclarations-manifestes à visée esthétique et sociétale ${ }^{2}$, et bien qu'encore aujourd'hui certains collectifs réunissant des acteurs de cette industrie produisent des manifestes qui remettent en cause les processus de production de la mode - délocalisation, industrialisation, fast fashion - de nouvelles pratiques surgissent et mobilisent ce que j'appelle le format-manif comme un prétexte communicationnel. Il m'a semblé intéressant de centrer l'étude sur ces formats-manif afin d'observer les pratiques de récupération du manifeste, les espaces dans lesquels il circule et se voit détourné et éventuellement les finalités de cette démarche.

2 Si en tant que production discursive en lien avec l'art ou la littérature le manifeste suscite, pour la recherche, des interrogations concernant son cadre générique et même, pour faire écho à la thématique de ce numéro, son caractère indiscipliné, dans ce qui suit, j'émettrai l'hypothèse que lorsqu'il est mobilisé à des fins marketing par l'industrie de la mode, le manifeste se comporte de façon très disciplinée. À partir de cette hypothèse j'emploie le terme de format-manif afin de souligner deux caractéristiques du corpus étudié. La première porte sur la nature standardisée et figée des formes communicationnelles proposées par l'industrie de la mode lorsque cette dernière s'empare des codes propres aux manifestes mais aussi aux manifestations. Le terme de format désigne donc cette standardisation. La deuxième caractéristique porte sur le paradigme étendu dans lequel l'industrie de la mode puise ses stratégies 
communicationnelles à positionnement revendicatif, voire subversif ${ }^{3}$. Des manifestes, mais aussi des manifestations, voire des messages à dimension contestataire et qui pourraient avoir leur place dans un manifeste ou dans une manifestation. Le terme de manif est alors employé dans la perspective rhétorique de la « litote», cette figure qui permet de dire "moins pour dire plus» (Groupe $\mu$ 1982: 133), désignant ainsi différentes pratiques présentant un point en commun : un discours pamphlétaire ${ }^{4}$.

Le corpus est composé de données hétérogènes pour ce qui est de leurs « contrats de communication» (Charaudeau 2005) et de leurs supports médiatiques, à savoir publicitaires, éditoriaux et marchands. Sont soumis à l'analyse :

- La campagne publicitaire de la marque de cosmétiques Lancôme pour le rouge à lèvres matte shaker, optant pour une mise en scène rappelant les codes gestuels que l'on trouve lors d'une manif (fig. 1).

- L'éditorial de mode intitulé Le Printemps. C'est la lutte des sacs!, paru dans le numéro $82 \mathrm{du}$ titre Citizen $K$ - printemps 2017, imitant une manifestation en plein air (fig. 2)5.

- Le défilé Chanel prêt-à-porter de la saison printemps-été 2015, présenté à Paris, au Grand Palais mettant en scène une manifestation ${ }^{6}$.

- La campagne publicitaire de la marque Yves Saint Laurent (dorénavant YSL) pour la collection automne 2008-hiver $2009^{7}$ proposée sous forme de tract-manifeste, diffusé à Paris, New York, Londres, Milan, Tokyo et Hong Kong et accompagné d'un bonus-cadeau, soit un tote bag (fig. 4 et 5).

- La campagne publicitaire consacrée au parfum Manifesto de la même marque, datant de 2012 (fig. 6).

- Le manifeste publié sur la plateforme numérique de la marque italienne Fendi intitulée $F$ is for... ${ }^{8}$.

4 Le point de vue développé tout au long de cette recherche est à la fois discursif, sémiotique et communicationnel. Discursif, dès lors que des notions ancrées dans l'analyse du discours sont mobilisées afin de démontrer la dimension figée du formatmanif. Sémiotique, dans la mesure où une analyse étayée de l'image permet de démontrer la mobilisation de signes propres au paradigme manif. Enfin communicationnelle, car la circulation et la récupération du manifeste permettent de saisir sa trivialité, cette dernière, définie par Yves Jeanneret comme "une catégorie descriptive " (2008: 14) observant la culture à travers «le fait que les objets et les représentations ne restent pas fermés sur eux-mêmes mais circulent et passent entre les mains et les esprits des hommes » (Ibid.).

\section{La manif comme spectacle médiatique : réinvestissements et détournements}

Relevant de l'espace public, la manifestation est ce rassemblement statique ou en mouvement réunissant un nombre relativement important de personnes en vue d'une protestation et d'une revendication à caractère social, politique, géopolitique, pacifique, etc. Cet événement peut être qualifié de spectacle dans la mesure où il est observé comme un élément discontinu et extraordinaire, invitant les spectateurs à focaliser leur regard dans ce qui se produit pendant ladite manifestation. Des codes informels mais qui ont tendance à se répéter constituent le langage d'une manifestation: pancartes et panneaux à messages, microphones, hauts parleurs accompagnent les manifestants dont la gestuelle peut également contribuer à la 
formation de ce langage, poings levés, postures dynamiques, visages expressifs. Si la rue est le médium d'une manif, la médiatisation de cette dernière au travers d'autres supports médiatiques tels que la photographie ou la vidéo contribue à la création du méta-spectacle, dans la mesure où ce dernier dépend du point de vue d'un observateur (photographe, journaliste, cameraman, etc.) permettant de saisir cet événement et éventuellement de le médiatiser.

6 Trois éléments du corpus proposent une mise en scène imitant les codes formels d'une manif: la publicité Lancôme, le défilé Chanel, et l'éditorial Le printemps. C'est la lutte des sacs! Dans ce qui suit, il s'agira de relever les signifiants transversaux à ces trois éléments, produits selon des procédés d'intertextualité et qui spectacularisent ${ }^{9}$ une manif.

Fig. 1. Publicité Lancôme, Matte Shaker

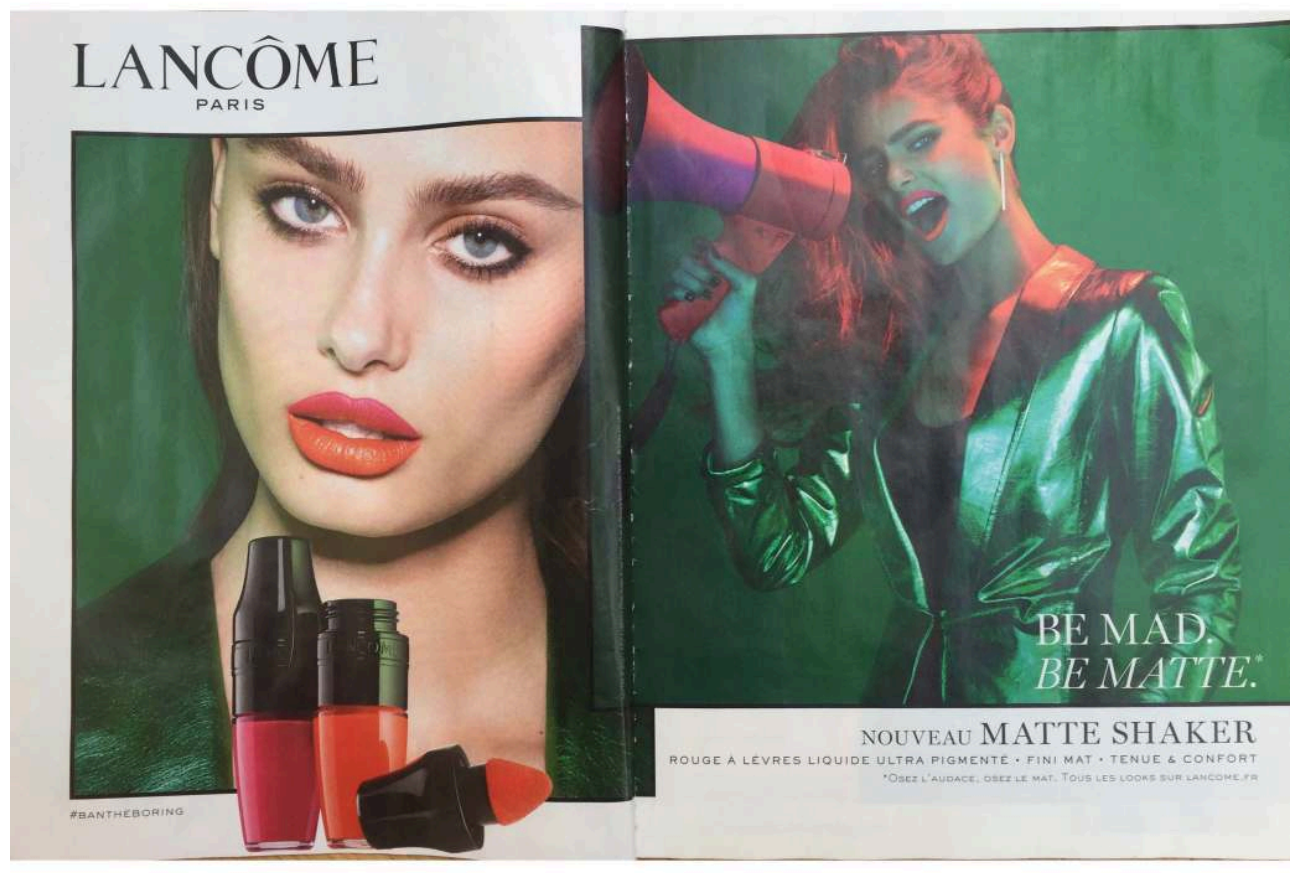

Publicité parue dans le numéro 446 du titre Biba en avril 2017, p. 6-7. L'image présentée ci-dessus est

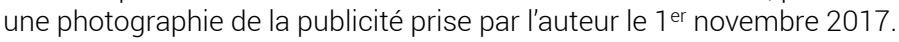

7 Proposée en double page, la publicité Lancôme présente sur la page de droite un mannequin debout tenant un haut-parleur; son expression faciale est dynamique et renvoie un état de colère, sa posture corporelle revendicative. Cette mise en scène construit l'image d'un personnage de manif, accompagné d'un message, un acte de langage directif ${ }^{10}:$ « Be mad. Be matte ${ }^{11}$.»

L'éditorial Le printemps. C'est la lutte des sacs! met en scène une situation conflictuelle se déroulant à l'extérieur et rappelant un certain désordre que l'on peut rencontrer lors d'une manif. Deux doubles pages et huit simples composent cet éditorial, construit comme un continuum intertextuel iconique et verbal. Pour des raisons d'économie, j'analyserai uniquement la première double page (fig. 2). Elle annonce le thème de l'éditorial dès son titre, formé à partir d'une commutation opérée au préalable sur la locution $^{12}$ lutte des classes et ayant donné lieu à l'énoncé la lutte des sacs. Quant à la mention temporelle fournie par cet éditorial, soit Le printemps, elle donne lieu à deux 
lectures possibles. La première renvoie à la segmentation des collections de la mode selon deux saisons principales ${ }^{13}$; la seconde renvoie aux qualifications de différentes révolutions politiques et sociétales et donc à d'autres locutions, telles que Printemps arabe ou Printemps de Prague.

Fig. 2. Éditorial paru dans Citizen $K$

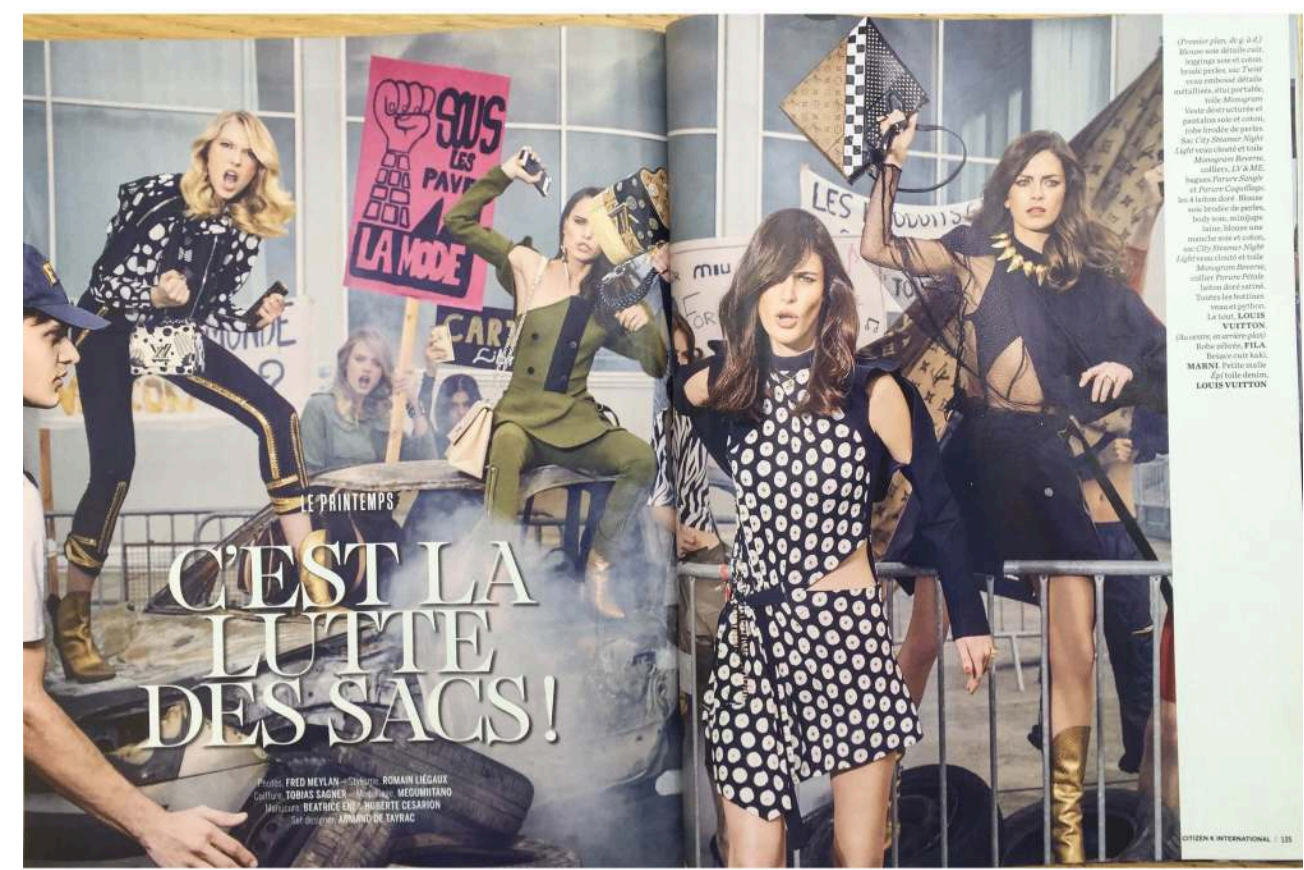

Éditorial paru dans le numéro 82 - printemps 2017 du titre Citizen K International, p. 134-135. L'image présentée ci-dessus est une photographie de la première double page de l'éditorial prise par l'auteur le $7^{\text {er }}$ novembre 2017.

La double page met en scène des actants animés et non animés dans une ambiance manif: des barricades en fer, des pneus dispersés, un véhicule carbonisé, de la fumée et de nombreux mannequins ${ }^{14}$. Les mannequins sont présentés de face, poing levé, hissant des pancartes à messages revendicatifs. Une grande écharpe aux motifs du logotype de la marque Louis Vuitton est également hissée et agitée, à l'instar d'un drapeau. Une pancarte rose présente l'énoncé sous les pavés la mode inscrit à côté du dessin d'un poing levé et d'une usine. Là encore, nous assistons à un détournement de l'énoncé sous les pavés, la plage, slogan ayant accompagné les luttes durant mai 1968, et dont la circulation pendant cette période a contribué à la formation de sa valeur symbolique ainsi qu'à son figement sémantique. Comme pour la locution lutte des classes, celle de sous les pavés la plage subit un défigement par son introduction dans l'espace communicationnel de l'éditorial de mode et du titre de presse spécialisée. La même opération de défigement peut également être observée pour les dessins proposés sur la pancarte. Le symbole du poing levé renvoyant, historiquement, à la lutte contre le fascisme et plus tard contre le racisme ${ }^{15}$ tandis que le dessin de l'usine symbolisant la lutte des classes ouvrières, leur introduction dans ce contexte communicationnel précis les prive de leur signification symbolique et les soumet à un nouveau processus de sémantisation. 
10 Autant la campagne publicitaire proposée par Lancôme, que l'éditorial de mode publié dans Citizen $K$ International peuvent être lus et interprétés comme un intertexte hyberbolique, soit un ensemble d' "échos libres d'un (ou de plusieurs) texte(s) dans un autre texte " (Adam 1999: 85) dont les signes sont exacerbés. D'une part, les signes constitutifs des deux discours - publicitaire et éditorial - sont là pour rappeler leur dimension fictionnelle dans la mesure où "aucun lecteur-consommateur ne croit vraiment au paradis-langage proposé par la publicité moderne ${ }^{16}$ " (Adam et Bonhomme 1997 : 54). D'autre part, les signes rappelant les manifs accordent à ces deux stratégies communicationnelles une dualité qui brouille les frontières entre fiction et réalité et tend à adopter une posture allant au-delà du publicitaire car optant pour un positionnement ancré dans le socle des luttes sociales. Le dernier exemple de ce procédé d'intertexte hyperbolique est le défilé manif proposé par la marque Chanel.

Fig. 3. Défilé Chanel, 2015, Grand Palais, Paris.

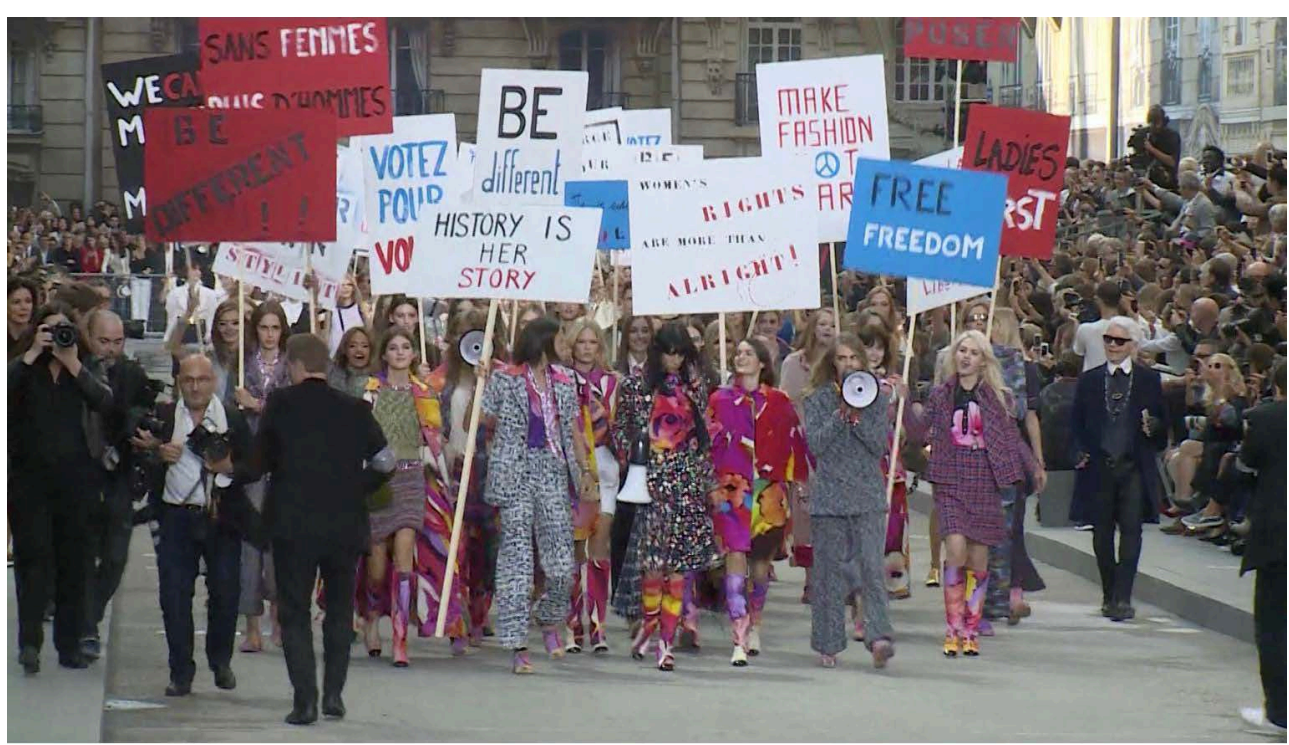

Copie d'écran effectuée à partir de la vidéo diffusée sur le site web : http://www.dailymotion.com/ video/x26yehx, consultée le 30 décembre 2017.

11 Sous la coupole du Grand Palais à Paris la collection printemps-été 2015 de Chanel a été présentée par des mannequins parcourant un boulevard nommé Chanel, entouré d'immeubles bourgeois haussmanniens. La fin de ce défilé se transforme en manif où l'ensemble des mannequins défilent, pancartes et haut-parleurs à la main, accompagnés de Karl Lagerfeld, directeur artistique de la marque. La gestuelle des mannequins est la même que celle observée pour les exemples précédents, à la différence que l'ambiance y est assez festive et joyeuse. Quant aux messages proposés sur les nombreuses pancartes du défilé manif, ils thématisent davantage la féminité, la liberté féminine, le rapport à la mode : be your own stylist, boys should get pregnant too, divorce pour tous, history is her story, ladies first. La spectacularisation de cet événement est particulièrement intéressante car elle relève du direct et se produit devant un public invité qui, comme l'image ci-dessus en témoigne, applaudit autant la collection de prêt-à-porter proposée par la marque que le spectacle ayant permis sa démonstration. 
12 Tous les éléments présentés ci-dessus s'inspirent d'un socle de codes culturels, dotés d'expressions sémiotiques plus ou moins figées, autrement dit, soumises à une représentation standardisée de la manif dans sa dimension organisationnelle que l'on peut rapprocher du stéréotype, défini comme « une représentation sociale, un schème collectif figé qui correspond à un modèle culturel daté » (Amossy et Herschberg Pierrot 1997 : 64). Formées à partir du principe de l'intertexte ${ }^{17}$, les trois manifs analysées détournent la manifestation dans son sens originel et la réinvestissent du point de vue de son inscription énonciative dans un espace médiatique dont la finalité est, du moins partiellement, commerciale. Observons comment ce réinvestissement s'opère et quelles en sont les conséquences.

13 Suivant Dominique Maingueneau, le processus de réinvestissement consiste, dans une perspective intertextuelle ${ }^{18}$, «moins à modifier qu'à exploiter dans un sens destructif ou légitimant le capital d'autorité attaché à certains textes [et] peut correspondre à deux stratégies opposées, la captation et la subversion » (1991: 155). La stratégie de la captation consiste en l'effacement du sujet parlant visant à «bénéficier de l'autorité attachée à ce type d'énonciation" (1991: 155), en l'occurrence celui du réinvestissement. Quant à la stratégie de la subversion, on est face à une tentative de "disqualification de cette structure dans le mouvement même de son imitation" (1991: 155). Les formats-manif observés présentent, à mon sens, la particularité de mobiliser autant le processus de captation et par là celui de la récupération d'une légitimité propre à la manif que le processus de subversion aboutissant à la disqualification de ses codes formels et de ses slogans symboliques. Comme si, dès lors que la manif a quitté la rue pour entrer dans un espace médiatique et pour servir des intérêts marchands - en l'occurrence issus de l'industrie du luxe - elle se privait de ses significations originelles et subissait un réinvestissement sémantique qui relève de ce que Maingueneau nomme la " parodie dévalorisante » (2002: 94).

Prenons comme exemple l'éditorial C'est la lutte des sacs! Comme énoncé en amont, les informations en marge de cette mise en scène indiquent que cette double page est entièrement consacrée à la marque Louis Vuitton. Par ailleurs, l'image même dévoile de façon ostensible des produits commercialisés par cette marque et dont l'utilité, dans ce contexte communicationnel précis, relève d'une double fonction: celle d'objet marchand mais aussi celle d'agent actif de la manif, comme c'est le cas de l'écharpe hissée tel un drapeau ou du sac à main brandi à l'instar d'une pancarte (cf. fig. 2).

Rappelons que la marque Louis Vuitton fait partie du groupe LVMH. Rappelons également que dans une optique contestataire, mettant implicitement en scène une certaine lutte des classes, elle-même issue des intérêts économiques en lien avec la commercialisation entre autres de sacs de luxe, le documentaire Merci Patron ${ }^{19}$ déploie la façon dont le groupe LVMH a contribué à la disparition de l'industrie du textile dans le nord de la France et par conséquent à des licenciements massifs des ouvrières et ouvriers du secteur. Il apparaît dès lors intéressant d'observer que l'énoncé lutte des sacs se construit à partir d'une locution, celle de lutte des classes dont la valeur symbolique est occultée et même remplacée par la répétition d'un signe marchand ostentatoire, le logotype de la marque Louis Vuitton. On assiste à un procédé de disqualification et de banalisation du langage (verbal, iconique et gestuel) à travers une mise en scène de détournement qui tend à réduire à un signifiant - dépourvu de signifié - toute forme langagière en lien avec la locution lutte des classes. Si nous 
acceptons, en suivant les travaux d'Alice Krieg-Planque, que cette locution fonctionne comme une formule $\left(2009^{20}\right)$, c'est tout son référent social qui est ici mis en cause.

\section{Le manifeste comme formule marketing: communication de marque et communication produit}

Le réinvestissement des codes propres aux manifestations permet de considérer les éléments ci-dessus observés comme des éléments triviaux soumis à un traitement "polychrésique ", qui selon Jeanneret consiste à "rendre compte du fait qu'une telle constellation d'objets [...] est exposée à faire l'objet de constantes réappropriations et à être ainsi prise sans cesse dans un large spectre de logiques sociales différentes " (2008: 83). Le deuxième exemple qui fait partie des formats-manif est celui du terme manifeste mobilisé en tant que titre de campagne publicitaire, en tant que nom de produit ou bien comme discours de marque.

Entre 2006 et 2011, la marque YSL ${ }^{21}$ a présenté les campagnes publicitaires de ses collections prêt-à-porter sous forme de manifeste, du moins pour ce qui est de son contrat de lecture et de son énonciation éditoriale (Souchier 2007). Pour cette étude, je me suis intéressée à la campagne 2008-2009 car elle introduit une nouveauté dans les modalités de distribution dudit manifeste. Proposé en exemplaires relativement limités, le manifeste était accompagné d'un tote bag YSL ${ }^{22}$. Il est dès lors important de souligner que ce manifeste se présente comme un produit organisé autour de son texte (le contenu du manifeste) et son paratexte (son emballage, en l'occurrence le tote bag et les modalités de sa diffusion). À l'instar de Gérard Genette pour qui le paratexte est « ce par quoi un texte se fait livre et se propose comme tel à ses lecteurs» (Genette 1987 : 7-8), la collection se donne à être lue à la fois comme un acte revendicatif, par son titre manifesto, et gratifiant, du fait de sa rareté (exemplaires limités, bonus cadeau inclus).

Pour ce qui est de la mise en scène scripto-iconique de ce document, l'on remarque, dès sa première page, sa dimension plastique ${ }^{23}$ (fig. 4 et 5 ) et plus précisément la façon dont l'image délimite et annonce son espace d'inscription. Le récit photographique n'attire pas particulièrement notre attention, dans la mesure où nous sommes face à une photo de mode assez canonique, thématisant la présence d'un mannequin qui prend la pose dans un espace intérieur. En revanche le contour de cette photo présente un double intérêt d'un point de vue sémiotique. Le premier est d'ordre plastique, le second d'ordre énonciatif. 
Fig. 4. Campagne publicitaire Manifesto YSL automne 2008 - hiver 2009

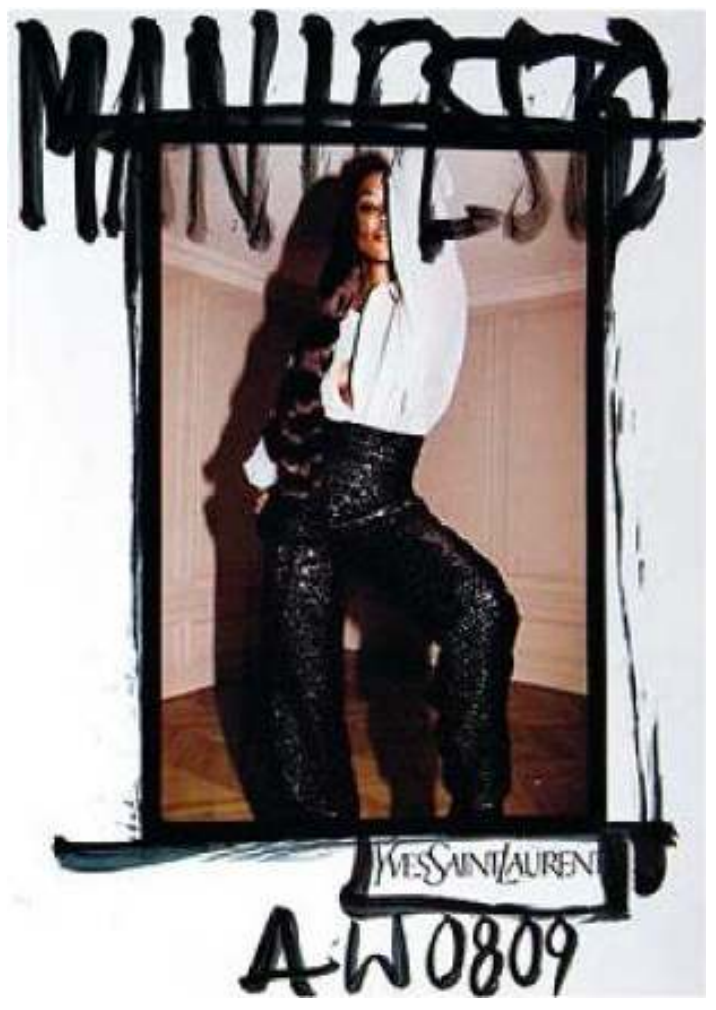

Copie d'écran effectuée à partir du site : http://www.hellocoton.fr/ysl-manifesto-feat-naomi-campbelldistribue-a-paris-le-6-sept-195695 (consulté le 30 décembre 2017). 
Fig. 5. Campagne publicitaire Manifesto YSL automne 2008 - hiver 2009 (tote bag)

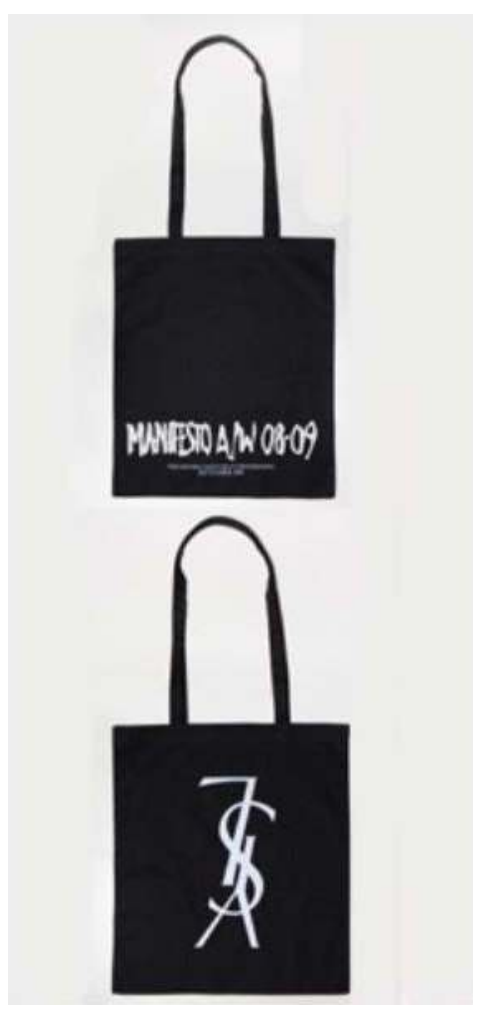

Copie d'écran effectuée à partir du site : http://www.vogue.fr/mode/news-mode/articles/troisimemanifesto-de-stefano-pilati-pour-ysl/17864 (consulté le 30 décembre 2017).

D'un point de vue plastique, les lignes délimitant la scène photographique rappellent, par leur organisation formelle (des traits hâtivement tracés), par leur qualité chromatique (un noir à la teinte irrégulière) et par leur texture (d'une certaine épaisseur), les inscriptions graffitis que l'on trouve dans l'espace public, inscriptions souvent liées à des revendications anarchistes. Elles peuvent également renvoyer à des tags et notamment à des inscriptions-détournements que l'on trouve sur des affiches publicitaires. Ce même effet plastique est observé pour l'inscription du Manifesto, titre de cette campagne publicitaire. Posée en haut de la photographie en guise de titre, ses lettres débordent le cadre et s'introduisent dans la scène photographique, comme si elles visaient à l'abîmer et la salir, tandis que, en suivant la même organisation plastique, la mention AW0809 est introduite en bas et à l'extérieur de l'image. Ce tracé contourne et encadre la mention de la marque productrice de cet événement photographique, posée en bas et à droite de l'image, mention inscrite, quant à elle, dans la police typographique habituelle du logotype de la marque.

L'organisation énonciative de cette image introduit des oppositions formelles produites par la corporéité explicite provoquée par l'effet graffiti ou tag et par l'exposition manuscrite des mentions Manifesto et AW0809. Ceci marque la présence d'un énonciateur autre que la marque ou les photographes ayant réalisé cette campagne publicitaire, soit une instance externe qui s'approprie la publicité de manière revendicative, autant sur le plan de la forme, par l'effet graffiti, que sur le plan du contenu, par les significations découlant du titre Manifesto. Bien que ce document génère des horizons d'attente précis, liés au statut de son émetteur explicite, une instance commerciale, et à son énoncé, une collection de prêt-à-porter, son intitulé et 
son effet plastique contribuent à transgresser et modifier ces attentes et partant, tout le contrat de lecture déterminé par cette campagne publicitaire.

Liée à la culture du graffiti, cette affiche publicitaire se dote d'une épaisseur discursive qui déploie la trivialité du manifeste, les traductions et même les détournements qu'il peut subir. Ici, le manifeste est intimement lié à l'acte du détournement, à la contestation du publicitaire même et dévoile un positionnement réflexif de la part de la marque. Comme si YSL visait non seulement la promotion de sa collection, mais aussi le développement d'un argument vis-à-vis des pratiques communicationnelles de l'industrie de la mode - ce qui demeure toutefois antinomique, car nous sommes face à un procédé qui ne fait qu'annuler la dimension axiologique du manifeste en tant que discours contestataire. Ce, du fait que la campagne publicitaire YSL mobilise la contestation comme un prétexte communicationnel, et que la dimension manifeste n'apparaît qu'au niveau de la forme et occulte toute démarche permettant de justifier ce choix énonciatif. Bien au contraire, la distribution en plein air de ce manifeste accompagné du sac en guise de cadeau consiste même à transformer le manifeste et toute la collection qu'il est censé représenter en prétexte permettant la distribution de ce bien YSL.

Une démarche relativement similaire pour ce qui est de la dimension plastique est observée dans la publicité proposée par YSL pour le parfum féminin nommé Manifesto.

Fig. 6. Publicité YSL pour le parfum Manifesto

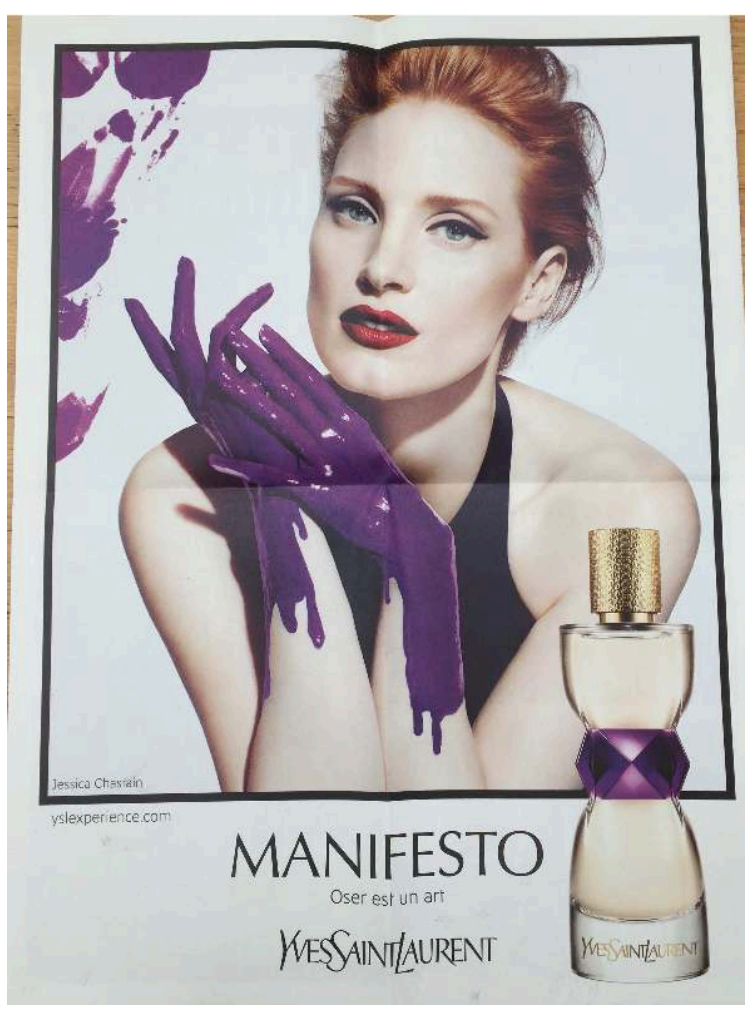

Photographie de l'auteur à partir d'un publi-communiqué réalisé par Vogue Paris pour Yves Saint Laurent beauté.

L'égérie de la marque pose sur un fond blanc investi par quelques traces de peinture d'une couleur violette très vive qui recouvre également ses mains. D'un point de vue sémiotique nous sommes face à une relation indicielle, construite sur le principe de 
cause à effet, entre la couleur violette et épaisse posée sur les mains de l'égérie et les traces de la même couleur et de la même matière visibles sur le fond blanc. On en déduit que ces traces sont produites par l'égérie de la publicité, jouant le rôle d'une artiste. Ce positionnement artistique est par ailleurs étayé par l'accroche inscrite endessous du nom Manifesto, soit: "Oser est un art». Comme pour la publicité précédente, le positionnement artistique semble accorder une certaine légitimité à l'emploi du terme manifesto, qui en l'occurrence est mobilisé pour qualifier un produit marchand. Nous assistons dès lors à une circulation du terme et par là à son détournement dès lors qu'il est mobilisé soit en tant que prétexte communicationnel, accompagnant une stratégie de publicité-produit soit en tant qu'appellation marchande.

Si l'on fait à nouveau appel à la notion de formule et si l'on considère, avec Alice KriegPlanque (2009 : 84), qu'elle est une forme discursive construite comme telle à travers ses usages sociaux, on peut constater que l'industrie de la mode procède à une traduction conséquente de la formule manifeste et par là à un tout nouvel usage social et plus précisément marketing. Il devient dès lors intéressant de saisir la non-étanchéité du terme manifeste, ce que je me propose de faire à travers le dernier élément du corpus. Il relève d'un format éditorial très proche du manifeste puisqu'il témoigne de sa forme déclarative en tant que texte écrit. Ce dernier cas permet d'interroger la circulation du terme lorsqu'il fait partie des stratégies discursives d'une entité commerciale notamment dans une optique de communication de marque.

Il s'agit d'un manifeste proposé par la plateforme numérique $F$ is for..., de la marque italienne Fendi ${ }^{24}$. Créée en 1925, la marque est initialement spécialisée dans le cuir et la fourrure et bénéficie pendant des décennies d'une clientèle particulièrement privilégiée mais aussi relativement âgée. Dans une optique d'extension de cible et de modernisation de la marque, Fendi crée en 2016 la plateforme $F$ is for... Si cet énoncé elliptique ouvre vers une multiplicité des choix permettant de commuter les points de suspension par un terme dont l'initiale serait la lettre $f$, soulignons que l'intertextualité de ce titre rappelle un autre euphémisme en anglais aussi revendicatif que trivial, à savoir $f$ is for fuck.

La plateforme $F$ is for... fait partie du site web commercial de la marque Fendi et est conçue à l'instar d'un blog de marque, soit un journal numérique qui consiste à présenter des articles illustrés en lien avec un positionnement précis mais sans pour autant évoquer explicitement les activités commerciales de la marque éditrice. Visant une cible relativement jeune, $F$ is for... développe des contenus en lien avec la culture urbaine, la musique, le sport, le voyage, etc. Écrit en anglais et intitulé Manifesto, ce texte est construit à partir d'une modalité discursive " élocutive " (Charaudeau 1992 : 574-575) - un on collectif - et adressé à une instance « délocutive » (574-575) construite à partir de l'adresse initiale - «dear world ${ }^{25} »$ - même si le texte laisse sous-entendre un profil de destinataire déduit. De manière générale, ce Manifesto construit un discours dialogique (Bakhtine et Volochinov 1977) durant lequel nous repérons des sousentendus, des clichés ou des stéréotypes vis-à-vis de l'instance productrice: "please stop calling us millenials ${ }^{26} »$. Titré freaks ${ }^{27}$ ce texte présente un discours réflexif qui est censé être écrit par un collectif de jeunes eux-mêmes représentés à travers les différentes images publiées sur cet espace numérique. Cette instance, bien que marquée par le pronom personnel du pluriel we, demeure in fine une parole déléguée : celle de la marque Fendi. En témoignent les nombreuses références faites à cette dernière à 
travers les différents contenus publiés, comme celui intitulé « $\mathrm{f}$ is for front row freaks ${ }^{28}$ » où il s'agit de la publication d'instantanés du défilé Fendi printemps-été $2018^{29}$.

Fig. 7. Header de la plateforme $F$ is for....

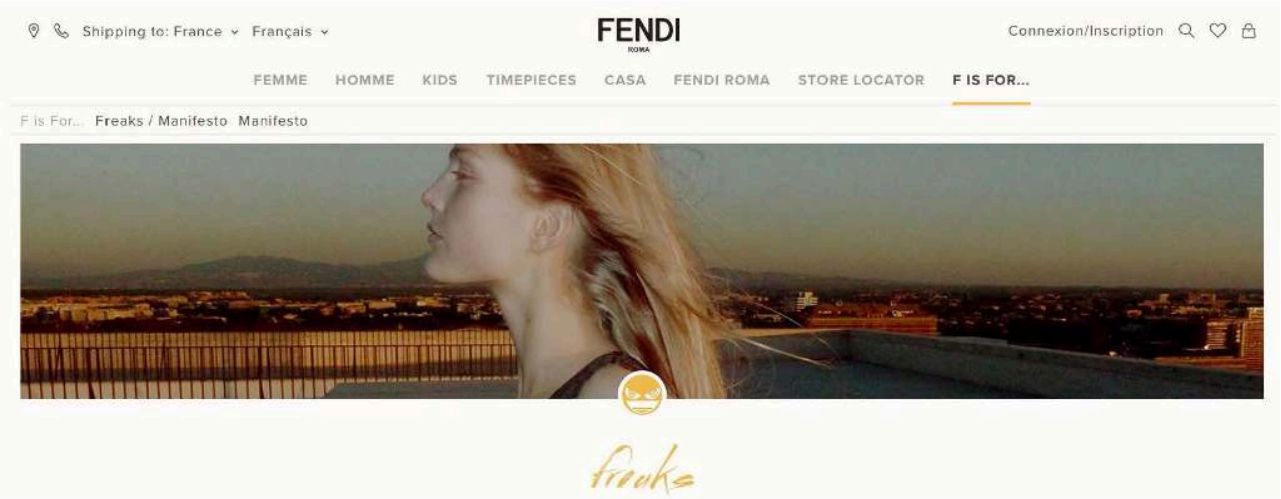

Copie d'écran effectuée à partir du site web de la marque Fendi : https://www.fendi.com/fr/fisfor/ categories/freaks/manifesto (consulté le 30 décembre 2017).

Fig. 8. Manifeste de la plateforme $F$ is for....

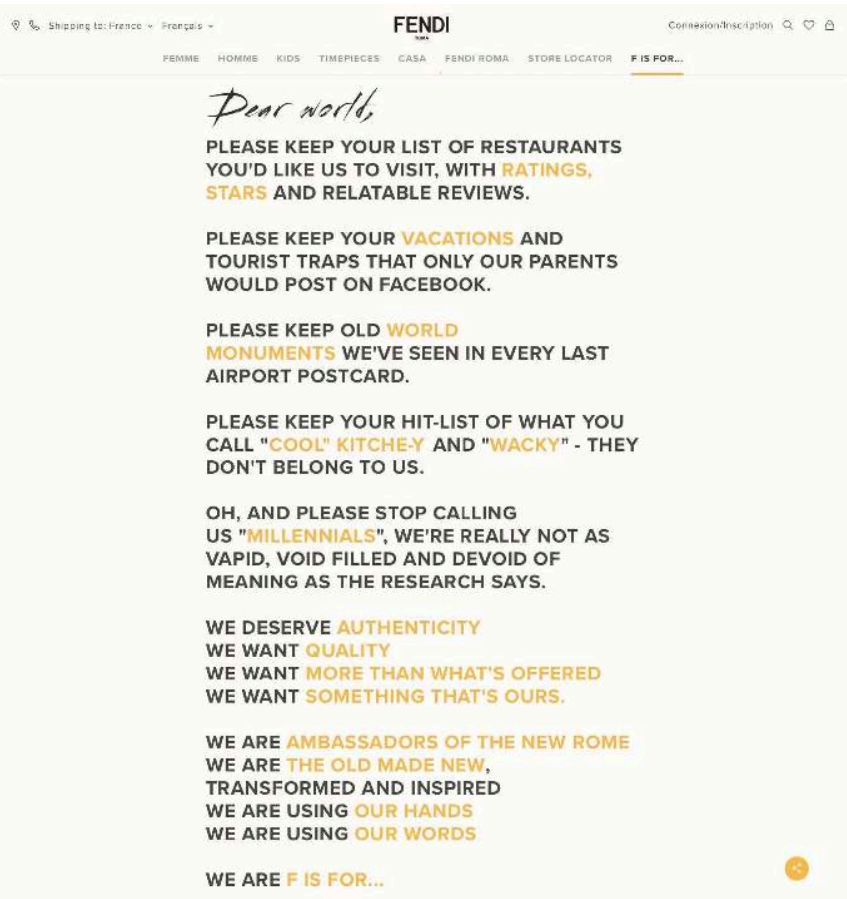

Copie d'écran effectuée à partir du site web de la marque Fendi : https://www.fendi.com/fr/fisfor/ categories/freaks/manifesto (consulté le 30 décembre 2017).

\section{Conclusion}

Au moment de conclure cette étude qualitative et non exhaustive des formats-manif de l'industrie de la mode, il paraît important de souligner deux points. Le premier porte sur l'attitude de récupération d'un discours revendicatif opérée par l'industrie de la 
mode et sur les enjeux qui déterminent cette attitude. Le second porte sur la dimension maniable du manifeste et par là sur son potentiel trivial.

Les formats-manif observés et analysés relèvent de façon explicite ou relativement implicite des processus de communication marchande et peuvent donc être pensés, à l'instar de la notion de pub ${ }^{* 30}$ proposée par Karine Berthelot-Guiet, Caroline Marti de Montety et Valérie Patrin-Leclère, comme un concept destiné :

[...] à saisir les évolutions de la publicité contemporaine. La publicité est [...] considérée dans une perspective large: l'acte de mettre en scène sur la place publique [et] reste l'expression prégnante des marques, y compris quand elles tiennent compte des représentations socioculturelles susceptibles de les fragiliser pour élaborer des formes de communication qui donnent l'impression de s'en distinguer. (Berthelot-Guiet, Marti de Montety et Patrin-Leclère 2013 : 55)

Il semble possible de considérer que le manifeste et ses dérivés sont mobilisés par l'industrie de la mode notamment pour leurs qualités socioculturelles: discours revendicatifs voire éthiques, postures militantes, prises de parole engagées. De là, tout en accompagnant ou en faisant l'objet de discours essentiellement publicitaires, ils peuvent, éventuellement, contribuer à masquer leur intention promotionnelle et consumériste.

Particulièrement controversée pour ses pratiques de production (délocalisation, exploitation de mains d'œuvres très bon marché), pour ses stratégies managériales (conditions de recrutement des mannequins défilant particulièrement sélectives et alarmantes), et pour les imaginaires qu'elle véhicule notamment autour des normes de beauté féminine (maigreur et éternelle jeunesse), l'industrie de la mode est contrainte à inventer des formes communicationnelles susceptibles de maquiller toutes ces visions dysphoriques. Le manifeste et de façon élargie les formats-manif étudiés ci-dessus témoignent donc de cette volonté de construire une face - au sens goffmanien du terme soit :

[...] la valeur sociale positive qu'une personne revendique effectivement à travers la ligne d'action que les autres supposent qu'elle a adoptée au cours d'un contact particulier. La face est une image de moi déclinée selon certains attributs sociaux approuvés, et néanmoins partageable, puisque, par exemple, on peut donner une bonne image de sa profession ou de sa confession en donnant une bonne image de soi. (Goffman $1974: 9$ )

Quant au manifeste lui-même et aux manifestations, il apparaît que leur maniabilité repose sur leur dimension stéréotypée construite à partir de discours médiatiques et de processus de médiatisation. Paradoxalement, plus une représentation sociale a tendance à se standardiser plus elle peut donner lieu à des détournements, des discours caricaturaux et des glissements sémantiques. Ayant transformé les manifs en événements particulièrement spectaculaires, les médias ont contribué à « surdéterminer [leurs] formes signifiantes » (Jeanneret 2008:87) et en fin de compte à transformer leurs représentations médiatiques en une sorte de ready-made mobilisable pour tout genre discursif et toute forme éditoriale. Ce constat n'annule pas évidemment l'imposture de l'industrie de la mode lorsqu'elle se positionne comme instance collective soucieuse d'une écologie sociétale ou professionnelle. 


\section{BIBLIOGRAPHIE}

Adam, Jean-Michel, 1999, Linguistique textuelle. Des genres de discours aux textes, Paris, Nathan.

Adam, Jean-Michel et Bonhomme, Marc, 1997, L'Argumentation publicitaire. Rhétorique de l'éloge et de la persuasion, Paris, Nathan Université.

Amossy, Ruth et Herschberg Pierrot, Anne, 1997, Stéréotypes et clichés, Paris, Nathan Université.

Angenot, Marc, 1995, La parole pamphlétaire. Typologie des discours modernes, Paris, Payot.

Bakhtine, Mikhail et Volochinov, Valentin, [1929] 1977, Le Marxisme et la philosophie du langage, Paris, Minuit.

Barrère Christian et Santaga Walter, 2005, Une économie de la créativité et du patrimoine : la mode, Paris, Ministère de la Culture.

Berthelot-Guiet, Karine, Marti de Montety, Caroline et Patrin-Leclère, Valérie, 2013, « Entre dépublicitarisation et hyperpublicitarisation, une théorie des métamorphoses du publicitaire », Semen, $n^{\circ} 36$, p. 53-68.

Burin, Philippe, 1986, « Poings levés et bras tendus. La contagion des symboles au temps du front populaire ", Vingtième siècle, revue d'histoire, vol. 11, p. 5-20

Charaudeau, Patrick, 1992, Grammaire du sens et de l'expression, Paris, Hachette.

Charaudeau, Patrick, 2005, Les médias et l'information. L'impossible transparence du discours, Bruxelles, De Boeck.

Genette, Gérard, 1987, Seuils, Paris, Seuil.

Goffman, Erving, 1974, Les Rites de l'interaction, Paris, Minuit.

Gross, Gaston, 1996, Les Expressions figées en français, Paris, Ophrys.

Groupe $\mu, 1982$, Rhétorique générale, Paris, Seuil.

Groupe $\mu$, 1992, Traité du signe visuel, Paris, Seuil.

Jeanneret, Yves, 2008, Penser la trivialité. Volume 1. La vie triviale des êtres culturels, Paris, Hermes Lavoisier.

Krieg-Planque, Alice, 2009, La Notion de " formule » en analyse du discours. Cadre théorique et méthodologique, Besançon, Presses Universitaires de Franche Compté.

Maingueneau, Dominique, 1991, L'Analyse du discours. Introduction aux lectures de l'archive, Paris, Hachette.

Maingueneau, Dominique, 2002, « Captation », dans P. Charaudeau et D. Maingueneau (dir.), Dictionnaire d'Analyse du Discours, Paris, Seuil, p. 92-94.

Martinet, André, 1985, Syntaxe générale, Paris, Armand Colin.

Searle, John, [1979] 1982, Sens et expression. Études de théorie des actes de langage, Paris, Minuit. Souchier, Emmanuel, 2007, « Formes et pouvoirs de l'énonciation éditoriale », Communication et langages, $\mathrm{n}^{\circ} 154$, p. 23-38.

Spitzer, Leo, [1949] 1978, « La publicité américaine comme art populaire », Poétique, n 34, p. 152-171. 
Toré, Gian Maria, 2001, « Pour une sémiologie générale du spectaculaire : définitions et questions ", Actes sémiotiques, n 114, [En ligne], http://epublications.unilim.fr/revues/as/1914, consulté le 1er novembre 2017.

\section{NOTES}

1. Pour Barrère et Santaga, la mode dépend autant de la créativité d'un couturier ou d'un designer que d'une entité plus globale, collective qui gère une marque. On parle donc de créativité managériale ou de management créatif permettant à l'industrie de la mode une gestion créative de ses stratégies médiatiques et transmédiatiques (cf. Barrère et Santaga 2005).

2. Notamment Le vêtement masculin futuriste de Filippo Tommaso Marinetti publié en 1909. Pour les manifestes contemporains, voir par exemple celui du collectif Front de Mode (http:// www.adriennebornstein.com/\#/front-de-mode/, consulté le 30 décembre 2017), collectif initié par la marque Sakina M'Sa (http://www.sakinamsa.com, consulté le 30 décembre 2017) et composé d'autres marques du secteur ou le manifeste de Maison Standards (http:// www.maisonstandards.com, consulté le 30 décembre 2017). Dans cette contribution, je n'interroge pas ces manifestes qui ne relèvent pas, de façon explicite et contextuelle, d'une stratégie marketing, bien qu'ils permettent aux différentes instances commerciales les ayant conçus de développer un positionnement certainement réflexif, mais aussi distinctif, vis-à-vis de la concurrence.

3. En italiques car il s'agit bien d'un positionnement d'ordre marketing. Dans ce cas de figure, le positionnement est un choix stratégique de mise en scène et ne reflète pas forcément la réalité axiologique de l'entreprise qu'il représente.

4. Voir Angenot (1995). La notion de discours est ici abordée dans son acception sémiotique et ne désigne pas exclusivement le langage verbal.

5. L'éditorial peut être consulté à partir du lien: http://www.lebook.com/creative/citizen-klutte-des-sacs-editorial-2017 (consulté le 30 décembre 2017). Bien que le titre Citizen $K$ ne relève pas de l'industrie de la mode mais de celle de la presse magazine, il est pertinent de souligner les interdépendances qui existent entre ces deux industries : la presse magazine est un espace de représentations et d'imaginaires liés à l'industrie de la mode tandis que cette dernière est la principale source de financement de la presse magazine.

6. Un extrait du défilé peut être visionné en suivant le lien : http://www.dailymotion.com/video/ x26yehx (consulté le 30 décembre 2017).

7. La marque se prénommait encore Yves Saint Laurent. Dans cet article il sera par conséquent question d'Yves Saint Laurent (YSL) et pas de Saint Laurent Paris.

8. https://www.fendi.com/fr/fisfor/categories/freaks/manifesto (consulté le 30 décembre 2017).

9. Je suis ici les travaux de Gian Maria Toré (2011) selon qui la spectacularisation « est un procès qui rend spectaculaire non seulement n'importe quel art, mais aussi n'importe quel autre domaine sociosémiotique ». Dans le cas du corpus étudié, la spectacularisation est double car elle met en scène un spectacle lui-même issu d'une convention, la manif dépendant de certaines règles de mise en scène. Ainsi, au-delà du constat que la spectacularisation est un processus inhérent au spectacle ordinaire, elle est ici saisie comme une exacerbation voire un commentaire d'un spectacle existant. Les images publicitaires ou le défilé de mode dépendent en effet de cette double spectacularisation car ils doivent introduire dans leurs mises en scène respectives autant leurs codes génériques originels que ceux issus du spectacle-manif.

10. Conformément à la théorie de John Searle, l'acte de langage directif fait partie des cinq catégories d'actes illocutoires et consiste à essayer « de faire faire des choses à autrui » ([1979] $1982: 32)$. 
11. Soit Soyez en colère. Soyez matte et contrairement à la traduction proposée par l'énonciation éditoriale de la publicité elle-même, traduction très nuancée : osez l'audace; osez le mat.

12. Selon Gaston Gross, une locution est un groupe dont «les éléments ne sont pas actualisés individuellement» $(1996: 14)$ et dans le cadre de laquelle «il ne peut pas avoir de relation prédicative entre les différents éléments qui sont dans la portée du figement » (1996: 15). Selon André Martinet et la syntaxe fonctionnelle, une expression figée fonctionne comme un synthème dont les éléments ne sont ni interchangeables ni en mesure de contracter «de relations particulières avec quelque autre monème extérieur [...] ; un chemin creux de fer ou un chemin de fer forgé ne serait plus un chemin de fer " (Martinet 1985 : 37). Qualifier l'énoncé la lutte des classes de locution ou de synthème permet de saisir et d'asseoir sa dimension figée et par conséquent non commutable et non modifiable, sans tenir compte bien évidemment de l'intervention de la créativité publicitaire. Comme l'indiquent Amossy et Herschberg Pierrot, ces énoncés figés peuvent être défigés « avec les jeux de mots, dans les titres de presse et les slogans publicitaires » (1997: 88).

13. Soit automne-hiver et printemps-été.

14. Pour cette double page, tous les mannequins sont vêtus, comme l'éditorial le mentionne en marge de la mise en scène photographique, exclusivement des articles commercialisés par la marque Louis Vuitton.

15. Voir par exemple Burin (1986).

16. L'expression de paradis-langage est empruntée à Leo Spitzer ([1949] 1978).

17. Intertexte autant verbal que non verbal, la notion de texte étant ici saisie dans son acception sémiotique.

18. Bien que Maingueneau parle dans ce cas de «procédé hypertextuel », je préfère maintenir le terme d'intertexte conformément à la définition proposée par Adam.

19. Réalisé par François Ruffin, sorti en février 2016. Référence faite au président-directeur général et propriétaire du groupe LVMH Bernard Arnault.

20. Je postule ici l'idée que les signes gestuels et corporels observés dans ces mises en scène ainsi que les dessins - usine, poing levé - fonctionnent également comme des énoncés figés et par là comme des formules. Cette notion est définie par Alice Krieg-Planque comme "l'ensemble de formulations qui, du fait de leurs emplois à un moment donné et dans un espace public donné, cristallisent des enjeux politiques et sociaux que ces expressions contribuent dans le même temps à construire » (2008: 7).

21. Marque appartenant au groupe Kering, particulièrement concurrentiel de LVMH.

22. Un tote bag est un sac rectangulaire en tissu. Celui proposé par YSL présentait la signalétique de la marque.

23. Au sens sémiotique du terme, à savoir un discours doté de formes iconiques, chromatiques et texturales (Groupe $\mu 1992: 186-252$ ).

24. Fendi appartient également au groupe LVMH.

25. Cher monde.

26. «Merci de ne pas nous appeler millenials ».

27. Monstres.

28. La lettre $f$ est pour les monstres du premier rang (d'un défilé).

29. https://www.fendi.com/fr/fisfor/categories/fulgore/F-is-For-Front-row-freaks (consulté le 30 décembre 2017).

30. L'astérisque est proposé par les auteures. 


\section{RÉSUMÉS}

Contrainte de développer des discours susceptibles de maquiller certaines valeurs dysphoriques associées à ses pratiques et son image, l'industrie de la mode opte souvent pour des tactiques communicationnelles relativement atypiques. La mobilisation du manifeste et de certains de ses dérivés comme des manifestations relève de cette stratégie qui consiste à récupérer des discours figés voire symboliques issus du politique ou de l'artistique et à les réinvestir à des fins communicationnelles et surtout marchandes. Cette récupération témoigne également de la dimension triviale du manifeste et par là de la construction des discours standardisés qualifiés de formats-manifs.

Facing several controversial situations, fashion industry is often obliged to develop communication strategies in order to dissolve negative representations related to its management. Advertising can be a positive partner as long as it proposes uncommon tactics. Representations of manifestos and protest marches is a part of the way the fashion industry seize upon fixed and symbolic discourses related to political or artistic positions while introducing them into communicational and consumerist goals. This sort of distortion reveals manifesto's trivial dimension and contributes to the construction of standard discourses qualified as manifesto-patterns.

\section{INDEX}

Mots-clés : stéréotype, figement, formule, réinvestissement, trivialité, publicité

Keywords : stereotype, fixed discourse, distortion, triviality, advertising

\section{AUTEUR}

\section{ELENI MOURATIDOU}

Université Paris 13, Labsic 